\title{
A Novel Color Image Fusion for Multi Sensor Night Vision Images
}

\author{
J.Jenitha Christinal \\ Department of Information Technology, \\ Karunya University \\ Coimbatore, Tamil Nadu, \\ India
}

\author{
T. Jemima Jebaseeli \\ Department of Information Technology, \\ Karunya University \\ Coimbatore, Tamil Nadu, \\ India
}

\begin{abstract}
In this paper presents a simple and fast color fusion approach for night vision images. Image fusion involves merging of two or more images in such a way, to get the most advantageous characteristics of each image. Here the Visible image is fused with the InfraRed (IR) image, so the desired result will be single, highly informative image providing full information. This paper focuses on color constancy and color contrast problem.

Firstly the contrast of the infrared and visible image is enhanced using Local Histogram Equation. Then the two enhanced images are fused in three compounds of a LAB image using aDWT image fusion. This paper adopts an approach which transfer color from the reference image to the fused image using Color Transfer Technology. To enhance the contrast between the target and the background, a scaling factor is introduced in the transferring equation in the b channel. Finally our approach gives the Multiband Fused image with the natural day-time color appearance and the hot targets are popped out with intense colors while the background details present with the natural color appearance.
\end{abstract}

Keywords: Image Fusion, Histogram Equalization, Color Contrast Enhancement, Color Transfer Technology.

\section{INTRODUCTION}

The IR image records the thermal radiations emitted by the objects in a scene and can be utilized to discover targets as it has better hot contrast and can present camouflaged targets. The visible image has much higher-frequency information on the background, which is essential for target localization and situation awareness in remote sensing. The IR image contains the information that is not available in the visible image. The IR reflectance of objects will be different from the visible light. A Fusion of IR and visible images with different contents could be utilized to enhance the image quality.

The image fusion method goal is for enhancing the interesting objects to be visible in thermal images against the visible image surroundings. The results can contain the IR band data highlighted with unnatural colors for better perception [1]. The next goal in multiband fused image is to create the colored night vision image. These false colored night images are more pleasing to look than the plain IR images. The color mapping technique produces the results that resembles slightly the natural coloring in the daylight [2].

The color level which human can distinguish is about a hundred times more than the gray level and many experiments shows that the color fusion may improve the feature contrast of the image, which allows for better scene segmentation and object detection $[4,5]$. Here is to enhance the false color image, we use the color transfer technology which will give the multiband fused image as the natural day-time color appearance and the hot targets pops out with intense colors while the background details present with the natural color appearance. So color fusion is becoming more and more important in the research field and a number of color fusion methods have been proposed.

The Multiband Fusion method for combining infrared image with a visible image concentrates heavily on the surveillance and remote sensing applications. The fusion goal in surveillance is to enhance the interesting objects visible in thermal images against the visible image surroundings. In remote sensing applications Multiband image data are fused to increase the spatial resolution and to improve the information representation [3].

\section{ANALYSIS ON MULTI-BAND FUSED IMAGE METHODS}

A survey has been done on a different multi-band fused image based on its image fusion method and color transfer method. This analysis help to get the detailed information on various procedures, algorithms, Color Space Transform, Histogram Analysis and accuracy of Segment Recognition.

A. Local-Coloring method: The Local-Coloring method [4] is based on Image Segmentation and Recognition and Local Color Transfer methods are used to enhance the color mapping effect. However, these methods are even more expensive than the global method since they are timeconsuming procedures such as Nonlinear Diffusion, Local Recognition, Local Comparisons and Image Segmentation.

B. Fast Natural Color Mapping Method: The Fast Natural Color Mapping method [5] consistently renders the MultiBand Night Vision Imagery in the natural colors. This method was implemented using standard color lookup table techniques to optimize the match between the false color fused image and the reference image. Once the lookup-table has been derived, the color mapping can be deployed to different multi-band image sequences with similar scenes.

C. One Color Contrast Enhanced Method: The One Color Contrast Enhanced Image Fusion method [6] introduces a ratio of local to global divergence of the IR image into the color transfer equations. As a result, both hot and cold targets are popped out, where hot target appears in intense red and cold targets appears in cyan.

D. EM Algorithm: In EM algorithm [7] the low frequency band image and high frequency band images are fused by Non-Subsampled Contourlet domain. Then the color transfer 
method is implemented using YUV color space to make the final fused image. It gives very abundant detail information and the color metric representation.

E. Adaptive Color Fusion Method: In adaptive color fusion method [8] both the Infrared and visible source images are fused using NSCT domain, so as to produce an intermediate fused gray scale image. Then it is mapped into the YUV color space to form a pseudo-color image. Finally, the Color Transfer technique is employed, to give the pseudo-color image with the natural color appearance.

F. Fast Color Contrast Enhancement method: In Fast color contrast enhancement method [9] visible and IR images are pre-processed using Local Histogram Equalization. Consequently, the two enhanced images are fused into the three components of a Lab image by means of a simple linear fusion strategy. Then the color transfer technology in simplified by the Lab color space. But it is different from the global statistic method, by means of transferring equation in the ' $b$ ' channel is amended by a stretch factor. It will change according to the distance between the current luminance value and the mean luminance value.

These surveys present different methods with its own strengths and weaknesses. The block diagram of Image Fusion for Night Vision Image is shown in Figure 1

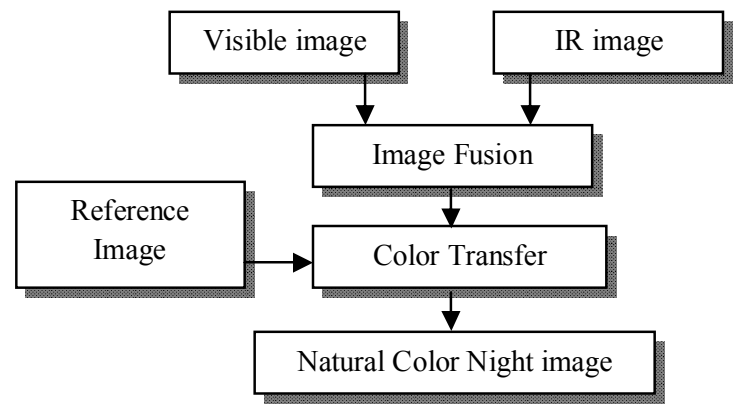

Figure 1 Block diagram of Image Fusion for Night Vision Image

\section{IMAGE FUSION}

The IR and visible light cameras have their own unique characteristics. Images taken in the visual spectrum tend to preserve good contextual information, while in night vision they usually show poor perception among objects due to the low contrast. IR images are almost insensitive to the change of light condition, so it may be more reliable to distinguish the targets from the background by the thermal contrast. To improve this, the contrast histogram equalization is done before the image fusion.

\subsection{Image Enhancement}

The proposed method consists of Histogram equalization; it's an effective method of improving the quality of low contrast images that is done by stretching the dynamic range of an image's gray level. This Histogram equalization method consists of global and local methods. Histogram equalization methods rescale the pixel values in an input image, to cover the entire available gray level image.

The contrast of an image is enhanced by the Global Histogram Equalization which is performed by enlarging the intervals of dense gray levels and also reducing those of sparse gray levels. The global Histogram Equalization method brings more noise if they occupy many numbers of pixels and other disadvantage is the images with extra high contrast make observer tired and anxious, which are not suitable for long time observation. To overcome these drawbacks, enhanced Visible and IR images were used.

In this method, First step is image segmenting, the input image is divided into several non-overlapping regions with the size of $5 \times 5$ and cumulative distribution function (CDF) is applied to the divided image, rather than the entire image. Then neighbouring regions are combined using bilinear interpolation to remove the artificially induced boundaries. The new pixel value is $f^{\prime}(i, j)$ at the position $(i, j)$ is[7]:

$$
\begin{aligned}
f^{\prime}(i, j)= & (1-u)(1-v) f(i, j)+(1-u) v f(i, j+1) \\
& +u(1-v) f(i+1, j)+u v f(i+1, j+1)
\end{aligned}
$$

where $f^{\prime}(i, j), f(i, j+1), f(i+1, j)$ and $f(i+1(j+1)$ are the values before interpolation in the enhanced image and $u, v$ are constant values $u, v \in[0,1]$

\subsection{Fusion of Infrared and Visible Image}

The enhanced Visible and IR image is fused by an Advanced Discrete Wavelet Transform (aDWT) method that incorporates the Principal Component Analysis (PCA) and Morphological Processing into a regular DWT fusion algorithm. The DWT method follows the multi-scale analysis method as same as the pyramid method. In DWT, the coefficients of two input images are fused pixel-by-pixel by choosing the average of the approximation co-efficients in the highest transform scale,in order to find largest absolute value of the detail co-efficients at each transform scale is processed. Then the inverse DWT is performed to obtain the fused image.

We incorporate Principal Component Analysis (PCA) and Morphological Processing into a DWT algorithm in order to obtain aDwt image fusion.

Step 1: To get DWT co-efficients of an image, the image is divided into four quarters: approximation detail, vertical detail, horizontal detail and diagonal detail.

Step 2: Apply PCA to the two input images approximation co-efficients at the highest transform scale that is by using the principal eigenvector derived from the two source images, as described below[6]:

$$
E_{F}=\left(a_{1} \cdot E_{A}+a_{2} \cdot E_{B}\right) /\left(a_{1}+a_{2}\right)
$$

where $E_{A}$ and $E_{B}$ are approximation co-efficients transformed from input images A and B. $E_{F}$ represents the fused coefficients, $a_{1}$ and $a_{2}$ are the elements of the eigenvector, which are computed by analyzing the original input images.

Step 3: Apply morphological processing, such as 'filling' and 'cleaning' operation to the image. Both filling and cleaning are the Matlab built-in morphological operation. Filling is used to fill the isolated interpixels and cleaning is used to remove the isolated pixels. These operations increase the consistency of co-efficient selection there by reducing the distortion in the fused image.

\section{COLOR TRANSFER}

In this proposed color transfer method, Color Based Clustering is applied on the Lab color space. Then Cluster Based Color Transfer is performed from natural color images using color similarity metric. The block diagram of the Color Transfer method is shown in Fig. 2.

The false color fusion can translate the gray information into easily distinguishable color information. Therefore 
combining all the bands in color space, it provides a method to increase the dynamic range of a sensor system. In the proposed method the false color fusion is performed in RGB color space. The false color fused RGB image can be represented by the following equations[11]:

$$
\begin{aligned}
& R(m, n)=1 / 2(\operatorname{vis}(m, n)+\operatorname{IR}(m, n)) \\
& G(m, n)=\operatorname{IR}(m, n) \\
& B(m, n)=|\operatorname{vis}(m, n)-\operatorname{IR}(m, n)|
\end{aligned}
$$

Here $m, n$ are the co-efficients values of each transforms. So the formed false color fused image has the intensity variations similar to the visible and IR source images. In order to achieve better separation in color based clustering, Decorrelation Stretch will be performed for color enhancement and Linear Contrast Stretch performed for intensity enhancement. Decorrelation Stretch increases the color separation across highly correlated channels by keeping the band variance as same. It is followed by a linear contrast stretch on individual RGB channels. The enhanced false color image obtained has more color variation and better contrast which significantly facilitates the subsequent clustering process.

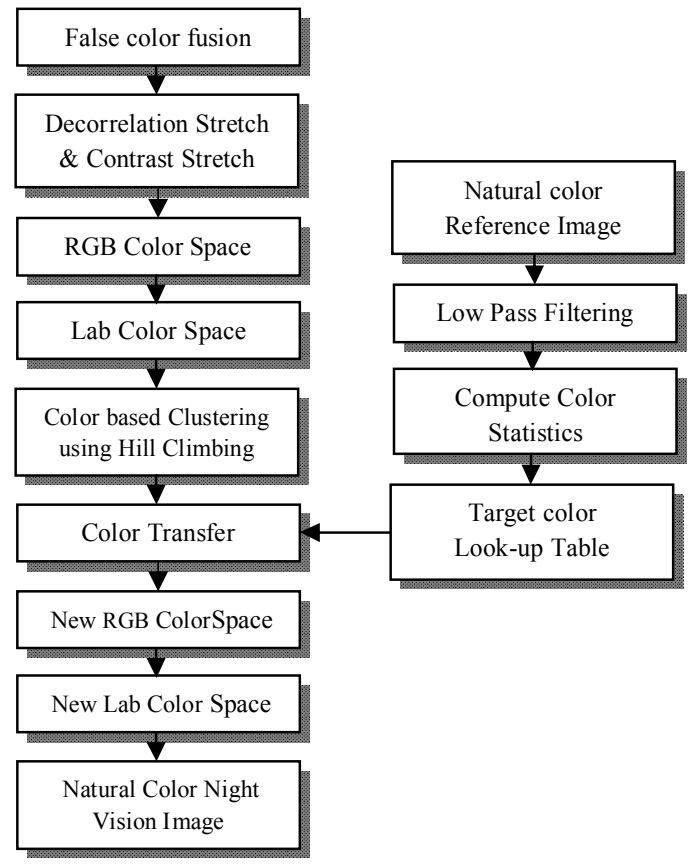

Fig. 2 Block diagram of the Color Transfer method for the Fused Image

\subsection{Color Based Clustering}

The enhanced false color fused image is converted to an indexed image where each pixel contains a single index which refers the RGB value in a color lookup table or color space. The RGB color space is then transformed into Lab color space to generate the Lab color space of the same size. Performing the color space transform decorrelates the three color components (i.e. L, a and b) so that manipulations such as statistic matching on each color component can be performed independently.

Color Based Clustering is performed on the Lab color space using the Hill Climbing Algorithm. A color based image segmentation method using Hill Climbing algorithm is utilized for Coloma clustering. The numbers of clusters required for proper classification of colormap are automatically determined by the Hill Climbing algorithm. The entries of the color space are then associated with the local maxima detected by the algorithm, to generate several coherent clusters in the Lab color space. The color transfer based on target color look-up table derived from the reference color images, gives the consistent color rendering and more natural appearance [4].

The target color look-up table is created as follows: From each image from the natural color target image is smoothed by the low pass filter and then transformed into the Lab color space and first order statistics, mean and standard deviation, are computed for each band.

\subsection{Color Transfer Technique}

Color transfer opertation is performed by cluster-bycluster using standard statistics matching method, Toet [5].

Then Lab color transformation is performed.

$$
\begin{aligned}
& L=116 \times f\left(Y / Y_{n}\right)-16 \\
& a=500 \times f\left(X / X_{n}\right)-f\left(Y / Y_{n}\right) \\
& b=200 \times f\left(Y / Y_{n}\right)-f\left(Z / Z_{n}\right)
\end{aligned}
$$

Here $X_{n}, Y_{n}, Z_{n}$ are the tristimulus values of reference white point, a line passing through an opposite color. Then the transformation is accelerated and normalized as follow: $X_{n}, Y_{n}$, $Z_{n}$ are the values used to normalize $X, Y, Z$ separately. In order to obtain the natural appearance, enhance the color transfer based on the color contrast.

Step 1: Subtract the mean value from the fused image data points, $(\mathrm{i}, \mathrm{e}$,

$$
\begin{gathered}
\tilde{L}=L-\left\langle L_{f u e}\right\rangle \tilde{a}=a-\left\langle a_{f u e}\right\rangle \tilde{b}=b-\left\langle b_{f u e}\right\rangle \\
\text { Here } L_{f u e}, a_{f u e}, b_{f u e} \text { is mean value of each channel. }
\end{gathered}
$$

Step 2: Then the data point are scaled with the help of the standard deviation of the fused image to the reference image.

$$
L^{\prime}=\frac{\sigma_{n, t}^{L}}{\sigma_{k, s}^{L}} \widetilde{L}+L_{f u e} \quad a^{\prime}=\frac{\sigma_{n, t}^{a}}{\sigma_{k, s}^{a}} \widetilde{a}+a_{f u e} \quad b^{\prime}=\frac{\sigma_{n, t}^{b}}{\sigma_{k, s}^{b}} \widetilde{b}+b_{f u e}
$$

where $\mathrm{k}^{\text {th }}$ cluster is associated with $\mathrm{n}^{\text {th }}$ natural image in the color look-up table, and $\mathrm{s}$ and $\mathrm{t}$ denote source and target respectively. The modified values of each transformation $L, a, b$ form a new transformation in Lab color space such that $L^{\prime}, a^{\prime}, b^{\prime}$.

\subsection{Enhancing the Target Detection}

After applying the color transformation to the fused image, the target detection is still ambiguous. The target detection cannot be achieved only by the intensity information in the color image fusion scheme.

Fortunately, the color contrast provides another cue in the color fused image, which makes the separation between the target and the background easier. So, the color image fusion should pop out targets in an instant color to improve their delectability.

To enhance the color contrast between the target and the background, we process each pixel in the blue - red channel with a stretch factor $w$ 


$$
b^{\prime}=w \cdot \frac{\sigma_{n, t}^{b}}{\sigma_{k, s}^{b}} \widetilde{b}
$$

Here in the IR image, the hot targets have larger luminance values. To maintain these characteristics, the stretch factor $w(i, j)$ of each pixel $(i, j)$ in the fused image is defined as, [4]

$$
\begin{aligned}
& w(i, j)=k \cdot \operatorname{dist}(i, j) /\langle\operatorname{dist}\rangle \\
& \langle\operatorname{dist}\rangle=\sum_{i=1}^{m} \sum_{j=1}^{n} \operatorname{dist}(i, j) / m \times n \\
& \operatorname{dist}(i, j)=\|L(i, j)-\langle L\rangle\|
\end{aligned}
$$

where $k$ is a constant that can be modified according to the actual requirement for the enhancement. $\operatorname{dist}(i, j)$ denotes the luminance divergence of the pixel $(i, j)$ from the mean intensity of $\mathrm{L}$ channel in the fused image. $L(i, j)$ and $m \times n$ are luminance value of the pixel $(i, j)$ and the size of the fused image separately.

As the hot target has great divergences to the mean intensity of L channel, it can be enhanced. Due to the color contrast enhancement, the hot targets are showed as intense red colors and the background are in natural color. Once the color processing is completed, the Lab color space is converted back to RGB color space.

\section{EXPERIMENTAL RESULTS}

The experimental result for UN camp image corresponding to the forest environment is shown in Fig.3. As shown Fig 3 (a) is a Visible image, in which the background is clearly showed, Fig 3(b) is an Infrared image in which source information cannot be detected, where the thermal information is visible. Both visible and infrared are the two input images. Fig 3 (c) is a reference color image of same size as the source image. Fig 3 (d) is a result of aDWT image fusion. Fig 3(e) is a result of the color transformation, where the color appearance indeed looks more natural, but the target can be hardly distinguished. Fig 3(f) the target is popped out with the intense red color. The average computational time to generate the color night vision image is $6-7$ seconds and to obtain the fused image is 3-4 seconds.

Entropy is used to measure the information content of an image. The entropy of the natural colored night vision image is computed for each band in RGB color space and the average of the entropy of the three bands is considered for evaluation

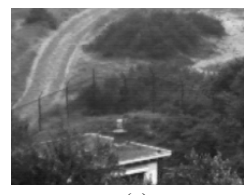

(a)

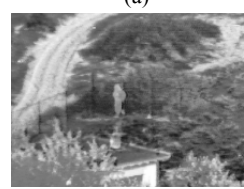

(d)

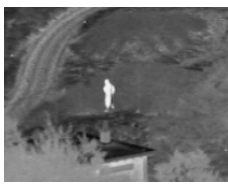

(b)

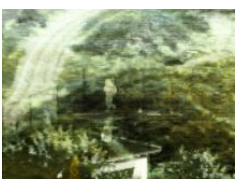

(e)

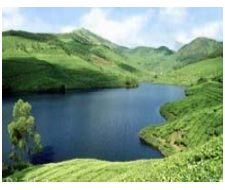

(c)

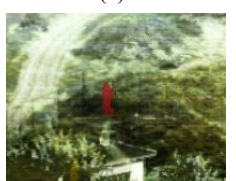

(f)
Fig 3 (a) Visible image (b)Infrared image (c) Reference image (d) aDwt fused image (e) Natural color image without target enhancement (f) Natural color image with target enhancement

\section{Fig. 3 Result of UN Camp Night Vision Image}

Colorfulness Metric is an efficient metric for calculating the colorfulness of the images; therefore the proposed method has the higher colorfulness metric. Finally the proposed method gives the Multiband Fused image as the natural daytime color appearance. From the proposed method the obtained entropy value and colourfulness metric value which is compared with statics matching method [4] and shown in Table 1.

Table 1. Comparison on Multi Band Fused Image

\begin{tabular}{|l|c|c|}
\hline \multicolumn{1}{|c|}{ Parameters } & $\begin{array}{c}\text { Statistics } \\
\text { Matching [4] }\end{array}$ & $\begin{array}{c}\text { Proposed } \\
\text { Method }\end{array}$ \\
\hline Entropy Value & 6.6375 & 6.7294 \\
Colorfulness Metric & 0.1852 & 0.3982 \\
\hline
\end{tabular}

\section{CONCLUSION}

In this paper, we proposed a simple and fast fusion approach for night vision image. Before fusion, the quality of both visible image and Infrared image is improved by using the Local Histogram equation and then the images are fused. To get the natural color image, the color is transferred from the reference image to the fused image by using Lab color space and the contrast between the target and the background is enhanced using a scaling factor. So the hot targets are popped out with intense red colors while the background details present with the natural color appearance.

\section{REFERENCES}

[1] A. Toet, J.I.J speert, A. Waxman, and M. Aguilar, "Fusion of visible and thermal imagery improves situational awareness," Displays, vol. 18, pp.85-95, 1997

[2] C. Pohl and J. L. van Gendern, "Multisensor image fusion in remote sensing concepts, methods and applications," International Journal of Remote Sensing, vol. 19, pp. 823-854, 1998.

[3] G.L. Walls. "The vertebrate eye and its adaptive radiation", Granbrook Institute of Science, Bloomfield Hills, Michigan, 2006.

[4] M.A. Hogervorst, A. Toet, "Fast natural color mapping for night-time imagery”, Information Fusion 11 (2010).

[5] A. Toet, "Natural color mapping for multiband night vision imagery”, Information Fusion 4 (2003)

[6] Y. Zheng and E. A. Essock, "A local-coloring method for night-vision colorization utilizing image analysis and fusion" Information Fusion, vol. 9, pp. 186 - 199, 2008.

[7] S.F. Yin, L.C. Cao, Y.S. Ling, et al., "One color contrast enhanced infrared and visible image fusion method", Infrared Physics and Technology 53 (2010) 146- 150.

[8] Gang Liu1, Guohong Huang2 "Color Fusion Based on EM Algorithm for IR and Visible Image” 2010 IEEE

[9] Weihua He, YongcaiGuo, Chao Gao "An adaptive color fusion method for night-vision images with NSCT" 2011 Elsevier.

[10] IshitMakwana, TanishZaveri and Vivek Gupta "Efficient Color Transfer Method based on Colormap Clustering for Night Vision Applica- tions " 2011 IEEE.

[11] "Night vision multiband source images data set available: http://www.imagefusion.org." 
International Journal of Computer Applications Technology and Research

Volume 2- Issue 2, 155 - 159, 2013

[12] M. A. Hogervorst and A. Toet, "Evaluation of a color fused dual-band nvg," International Conference on Information Fusion,

[13] R. S. Blum and Z. Liu, "Multi-sensor image fusion and its applications," Taylor \& Francis, CRC Press, 2006.

[14] D. Hasler and S. Suesstrunk, "Measuring colorfulness in natural images," Proc of Human Vis and Elect. Image. VIII. Santa Clara, CA, USA, 2003. 